\title{
Taurine in health and diseases: consistent evidence from experimental and epidemiological studies
}

\author{
Yukio Yamori, Takashi Taguchi, Atsumi Hamada, Kazuhiro Kunimasa, Hideki Mori, Mari Mori \\ From $17^{\text {th }}$ International Meeting of Taurine \\ Fort Lauderdale, FL, USA. 14-19 December 2009
}

\section{Abstract}

Taurine (T) was first noted as beneficial for stroke and cardiovascular diseases (CVD) prevention in genetic rat models, stroke-prone spontaneously hypertensive rats (SHRSP). The preventive mechanisms of T were ascribed to sympathetic modulation for reducing blood pressure (BP) and anti-inflammatory action. Recent epidemiological surveys revealed the involvement of inflammatory mediators in the pathogenesis of stroke and also atherosclerosis for which T was proven to be effective experimentally. Arterio-lipidosis prone rats, a substrain of SHRSP selectively bred for higher reactive hypercholesterolemia, quickly develop not only arterial fat deposition but also fatty liver which could be attenuated by dietary T supplementation. CARDIAC (CVD and Alimentary Comparison) Study was a WHO-coordinated multi-center epidemiological survey on diets and CVD risks and mortalities in 61 populations. Twenty-four-hour urinary (24U) T was inversely related significantly with coronary heart disease mortality. Higher 24U-T excreters had significantly lower body mass index, systolic and diastolic BP, total cholesterol (T-Cho), and atherogenic index (Al: T-Cho/high density lipoprotein-cholesterol) than lower T excreters. T effects on CVD risks were intensified in individuals whose $24 \mathrm{U}-\mathrm{T}$ and -magnesium (M) excretions were higher. Furthermore, higher $\mathrm{Na}$ excreters with higher heart rate whose BP were significantly higher than those with lower heart rate were divided into two groups by the mean of $24 \mathrm{U}-\mathrm{T}$, high and low T excreters. Since the former showed significantly lower BP than the latter, T may beneficially affect salt-sensitive BP rise. Included among the typical 61 populations, were Guiyang, China or St. John's, Newfoundland, Canada where in which the means of both 24U-T and -M were high or low, respectively. The former and the latter had low and high CVD risks, respectively. Australian Aboriginals living at the coastal area in Victoria were supposed to eat T- and M-rich bush and sea foods and be free from CVD 200 years ago, but they presently have nearly the highest CVD risks indicating that T- and/or M-containing seafood, vegetables, fruits, nuts, milk, etc, similar to prehistoric hunters' and gatherers' food should be good for CVD prevention. The preventive effects of T, good for health and longevity, first noted experimentally, were also proven epidemiologically in humans.
\end{abstract}

\section{Introduction}

The health effects of taurine (T), "a wonder molecule", was first noted in our genetic rat models for hypertension (Spontaneously Hypertensive Rats: SHR) [1,2] and stroke (Stroke-prone SHR: SHRSP) [3,4] and are now being confirmed epidemiologically by our WHO-

\footnotetext{
*Correspondence: yamori@cardiacstudy.com

Institute for World Health Development, Mukogawa Women's University, Nishinomiya 6638143, Japan

Full list of author information is available at the end of the article
}

coordinated Cardiovascular Diseases and Alimentary Comparison (CARDIAC) Study covering 61 populations in the world [5-8]. Advances in extensive studies on experimental models indicate that $\mathrm{T}$ is preventive against hypertension [9], stroke and atherosclerotic arterial diseases [10-12]. $\mathrm{T}$ is also expected to be effective for the prevention of nonalcoholic steato-hepatitis (NASH) increasing now in developed countries [13]. Accumulating evidence from CARDIAC Study indicates that common $\mathrm{T}$ intakes reduce cardiovascular disease

\section{Biomed Central}


(CVD) risks and contribute to the longevity of the Japanese [12] which have the lowest coronary heart disease (CHD) mortality in developed countries. Furthermore, the recent health study has revealed that CHD risks are definitely lower in the individuals and populations with higher 24-hour urinary (24U)-T and -magnesium (M) excretions [14].

\section{1) Taurine (T) and prevention of cardiovascular diseases (CVD)}

Since SHRSP was established as a genetic model developing stroke, extensive nutritional and pharmacological studies have been conducted experimentally $[7,9,10,15]$. Our experiments to feed SHRSP on high or low fish protein diets with or without $1 \%$ salt in drinking water first demonstrated that fish protein rich in $\mathrm{T}$ attenuated salt-induced severe hypertension and decreased stroke incidence from $80 \%$ down to $10 \%[4,15]$. High fish protein diet with low salt in drinking water was proven to be most effective to reduce stroke down to $0 \%$. We further investigated the effects of several amino acids contained in fish protein in SHRSP and confirmed that $\mathrm{T}$ is effective for reducing blood pressure (BP) in SHRSP [9]. Among our experiments to prove the effect of various nutrient and amino acid supplementations on BP and stroke incidence in SHRSP, sulfur amino acids, such as $\mathrm{T}$ and methionine, were effective on the reduction of BP and stroke incidence [6]. Other than sulfur amino acids reducing $\mathrm{BP}$, lysine had no effect on BP but was also effective for the prevention of stroke. We speculate therefore that enough amino acid supply to the vascular wall also might be protective against stroke $[6,15]$.

Therefore, we further analyzed the nutritional mechanisms of stroke in SHRSP and also in humans. The common predilection site of stroke is the basal ganglia which are fed through perforating arteries branching recurrently from the cerebro-basal arteries. Blood flow through such recurrent arteries is reduced hemorheologically in hypertension, and thus vascular damage develops due to the reduced blood supply to these arterial walls $[15,16]$. Anatomically the vascular wall of these recurrent arterial branches should receive nutritional supply only from the blood stream inside the vessel through the blood-brain barrier, whereas the cerebro-basal arteries receive nutritional supply through capillaries around the vascular wall, ("vasa vasorum"). Our morphological electron microscopic studies in 1980 's for the first time demonstrated that vascular damages start at the outer layer of the vascular smooth muscle cells located at the furthest site from the vascular lumen $[15,16]$. Moreover, we proved that macrophages were activated in response to the vascular damages that occurred initially at the outer layer of perforating arteries in the brain. Such nutritional vascular damages are followed by inflammatory reactions. At the advanced stage vascular damages occur at the inner layer of cerebral vessels which leads to vascular wall rupture that causes cerebral hemorrhage or to thrombosis inside the damaged blood vessels that causes cerebral infarction $[15,16]$. These pathological processes were further confirmed in human autopsy materials by an immuno-histochemical technique [17] in which macrophages were recruited to cause and heal cerebro-vascular damages. Vascular smooth muscle cells initially remained intact near the intima close to the vascular lumen as in SHRSP. Therefore, we can speculate that the activation of macrophages and other inflammatory cells are supposed to further damage cerebral arteries, causing severe vascular lesions such as angio-necrosis. However, when these inflammatory cells contain much $\mathrm{T}$ from nutrients, $\mathrm{T}$-chloramine neutralizes the adverse action of radicals produced from chlor [18] and may help vascular cells survive or regenerate to repair the wall [19].

After our demonstration of the importance of inflammation in stroke in rat models, the pathogenic concept of stroke as well as atherosclerosis in humans has also changed greatly [20]. High sensitive C-reactive protein (CRP), a marker and product induced by inflammatory reaction, was confirmed to be the reliable predictor of ischemic stroke and atherosclerotic CHD by extensive cohort studies $[21,22]$. Therefore, the control of local inflammation by $\mathrm{T}$ appears to be important for the prevention of stroke. The effect of fish protein on the incidence of stroke was experimentally first demounted in SHRSP in the 1970's $[4,15]$, and the cod protein feeding was reported to be clinically effective for reducing CRP in humans [23]. $\mathrm{T}$ or sulfur amino acids rich in fish protein may protect cerebral vessels through its anti-inflammatory action by the local formation of T-chloramines. Consistent with these studies, our world-wide epidemiological study showed that among 5 diet-related factors $\mathrm{T} /$ creatinine $(\mathrm{Cr})$ ratio was proven to be a potent preventive factor against stroke as well as CHD as mentioned later [12].

\section{2) Taurine (T) and lipotoxicity in the vascular wall and liver}

By our further effort for establishing a better model of atherosclerosis from SHRSP, the selective sib-mating of the substrain of SHRSP, developing greater reactive hypercholesterolemia in response to high fat cholesterol (HFC) diet feeding, was started in 1972 [24]. The strain developed not only reactive hypercholesterolemia but also ring-like fat deposits in small mesenteric arteries within 2 or 3 weeks on HFC diet. This appeared to be a good model for studying the initial process of fat 
deposition on arterial walls in the development of atherosclerosis [25] because similar ring-like fat deposits were noted at autopsy in the atherosclerotic lesions of cerebro-basal arteries in the human brain.

Dietary $\mathrm{T}$ supplementation was proven to be effective for attenuating the development of hypercholesterolemia and for decreasing fat deposits in the mesenteric arteries of the selected SHRSP fed on HFC diets [26,27]. Such cholesterol-lowering effect was also confirmed in volunteer medical students who took $6 \mathrm{~g}$ of $\mathrm{T}$ per day for 3 weeks [28]. These beneficial effects of $\mathrm{T}$ on hypercholesterolemia and arterial fat deposits were experimentally confirmed to be due to the increased bile acid production and the activation of $7 \alpha$-hydroxylase [26], the rate limiting enzyme in the process of the conversion of cholesterol into bile acids. This has been proven to be due to the enhanced gene expression of cholesterol $7 \alpha$ hydroxylase [29]. When T feeding lowered cholesterol levels, the gene expression was proportionally accelerated in relation to the reduced cholesterol level. Moreover, $\mathrm{T}$ supplementation was proven to effectively increase low density lipoprotein (LDL) receptors in the liver by an experiment of $\mathrm{T}$ supplementation accelerating the decay curve of radio-labeled (125I) LDL [30]. Therefore, cholesterol-lowering mechanisms of $\mathrm{T}$ are summarized to be due to an increase in LDL receptors and accelerated conversion into bile acids by $\mathrm{T}$, and other mechanisms such as T's effect to reduce intestinal acyl-CoA:cholesterol acyltransferase activity [26,31].

We are presently interested in the activation of LOX1 , a receptor for oxidized LDL that was up-regulated in SHRSP fed on HFC diet [32]. T actually decreased arterial fat deposition [26]. Therefore, $\mathrm{T}$ is speculated to attenuate the increase of LOX-1 receptors though a possible local antioxidant action by $\mathrm{T}$-chloramine formation $[19,31]$. The suppressive effect of $\mathrm{T}$ on LOX-1 was noted actually in experimental diabetic or hypertensive models treated by $\mathrm{T}[33,34]$.

In relation to arterial lipid deposition we could establish a new model for NASH [35], now increasing in Japan as in other developed countries because of the westernization of dietary habit to eat more fatty meat and less fish. The substrain of SHRSP successively bred by checking greater reactive hypercholesterolemia and rapid arterial fat deposition for the last 37 years was named "Arterio-lipidosis prone rats" (ALR, SHRSP 5/ Dmcr) [36]. The recent studies are confirming that ALR should also be a good model for NASH, developing not only fatty liver but also inflammatory reaction and active fibrosis forming pseudo-lobule as well as the typical morphology of Mallory's hyaline bodies within 8 to 12 weeks of HFC diet feeding (Figure 1). Dietary T's effect in this new model for NASH is expected, because subcutaneously injected $\mathrm{T}$ was reported to decrease serum lipid levels and serum transaminase levels, markers of hepatocyte damage as well as the biomarkers of oxidative stress in the rats fed on HFC diets developing fatty liver. Moreover, $\mathrm{T}$ suppressed hepatic mRNA expression of cytokines such as tumor necrosis factor-alpha and transforming growth factor-beta, possibly involved in the process of hepatocyte damage and fibrosis, whereas it increased the expression of a beneficial cytokine, adiponectin. However, the reported models are basically models for fatty liver and appear morphologically not to be typical models for NASH in humans as ALR. Since T supplementation was observed to decrease serum cholesterol levels and arterial fat deposition in ALR [36], if $T$ could also improve ALR's typical morphology of $\mathrm{NASH}, \mathrm{T}$ would be "a wonderful molecule" for nutritionally protecting CVD risks and $\mathrm{NASH}$, a common pathology in metabolic syndrome, and would therefore be expected to contribute to a healthy longevity in man.

In conclusion, our experimental studies consistently indicate greater prospective for $\mathrm{T}$ to contribute to the nutritional prevention of CHD, as well as stroke and probably $\mathrm{NASH}$, the increasing health problem of mankind exposed to urbanization and westernization of dietary habit and life styles.

\section{3) Taurine ( $T$ ) and coronary heart diseases (CHD) 3-1) T's role in CVD prevention}

These experimental findings to support the beneficial effect of $\mathrm{T}$ and other nutrients on CVD prevention led Y. Yamori to propose an international cooperative study on the association of dietary factors with CVD risks and CVD mortality in 1982. The protocol of this study obtained an international consensus of two meetings organized by WHO-Collaborating Center for Primary Prevention of CVD in 1983 and 1985 [5-7]. The CARDIAC Study is a multi-center cross sectional epidemiological survey for which about 100 males and 100 females aged 48 to 56 were randomly selected from each population and invited to participate in a health examination. This consisted of weight, height, BP measurement by an automated BP measurement system, blood tests for serum total cholesterol (T-Cho) and high density lipoprotein-cholesterol (HDL), as well as an analysis of biomarkers (the daily excretions and their ratios to $\mathrm{Cr}$ ) of $\mathrm{Na}$, potassium (K) M, T, isoflavones and urea nitrogen for estimating dietary intakes of salt, vegetables, milk products, seafood, soybean products and protein by sampling $24 \mathrm{U}[5-8,37,38]$. The associations of nutrition with BP and with CVD mortalities were analyzed in "Core" Study and in "Complete" Study, respectively. "Core" Study demonstrated that the population averages of BP, both systolic and diastolic BP (SBP and DBP), were positively associated with the average population intake of daily $\mathrm{Na}$ estimated by $24 \mathrm{U}-\mathrm{Na}$ excretion 


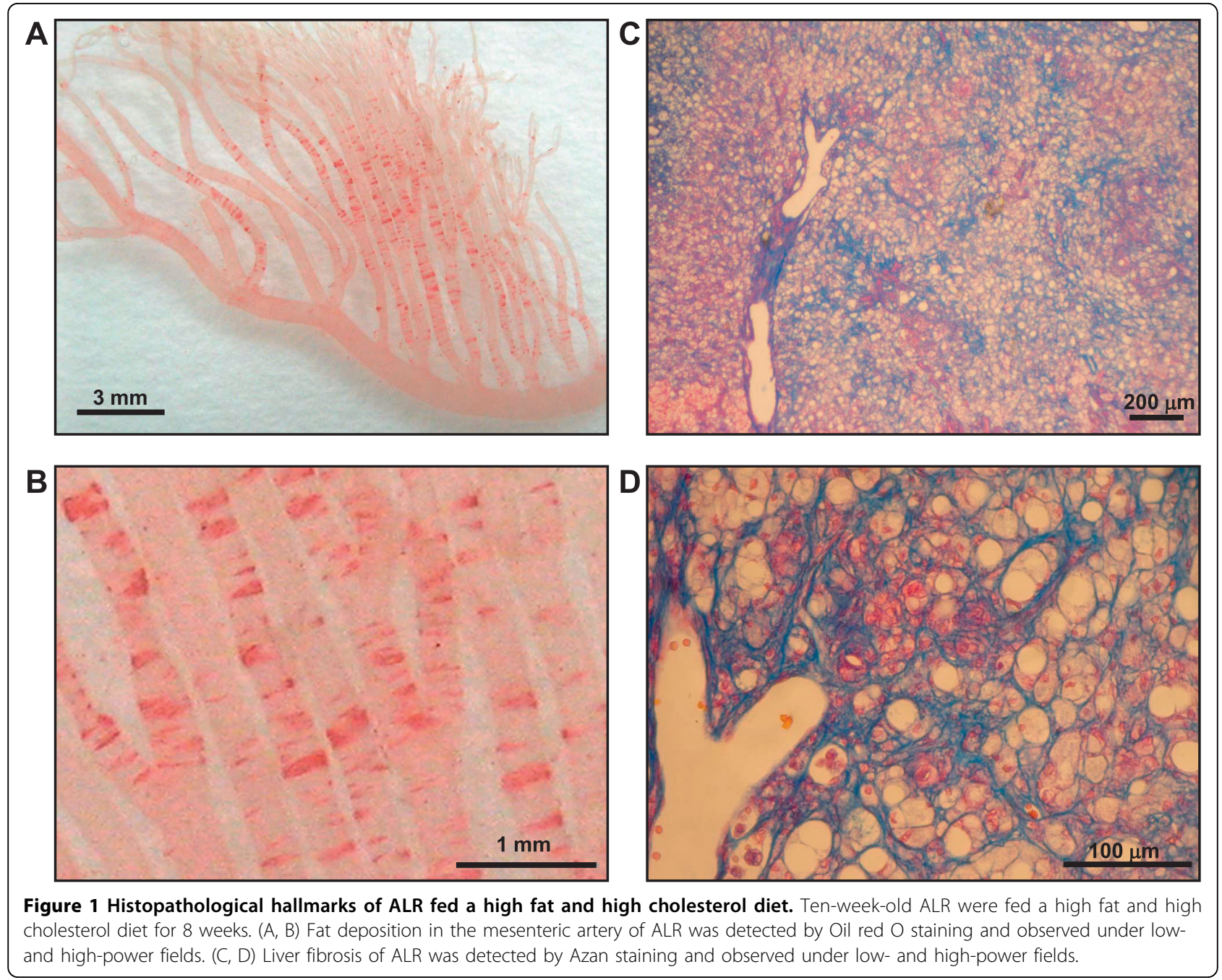

$[7,37,38]$, and the population average of $\mathrm{M} / \mathrm{Cr}$ in $24 \mathrm{U}$ were inversely associated with SBP and DBP indicating a significant association with DBP [7]. "Complete" Study showed significant positive association of the ageadjusted mortality rates of stroke with the population averages of $24 \mathrm{U}-\mathrm{Na}$ excretion or $24 \mathrm{U}-\mathrm{Na} / \mathrm{K}$ ratio, and with the arachidonic acid ratio in the plasma phospholipids [8], but the rates were significantly inversely related with T-Cho. Moreover, CHD mortality rates were significantly positively related with the population averages of serum $\mathrm{T}$-Cho and inversely related with polyunsaturated/saturated fatty acid ratios, n-3 fatty acid ratios of plasma phospholipids or $24 \mathrm{U}-\mathrm{T}$, indicating a protective effect of seafood intake on CHD $[8,37]$.

In CARDIAC Study populations we examined 5 dietrelated markers in relation to CHD by applying the structural equation modeling for males and females. Five markers were T-Cho, BMI and $\mathrm{Na} / \mathrm{Cr}, \mathrm{M} / \mathrm{Cr}$ and $\mathrm{T} / \mathrm{Cr}$ ratios in $24 \mathrm{U}$ (Figure 2) [12,38]. The results clearly indicated that higher $\mathrm{T} / \mathrm{Cr}$ ratios were highly significantly related to the risk reduction of CHD both in males and females, in contrast to T-Cho increasing the risk. Interestingly, our results first demonstrated $M$ was also strongly related to the risk reduction following $\mathrm{T}$. Since $\mathrm{M}$ content of seafood is also high, $\mathrm{T}$ and $\mathrm{M}$ are positively associated with each other. Twenty-four-hour $\mathrm{U}-\mathrm{T}$ excretions were widely distributed in 61 populations' averages, positively associated with seafood intakes and exceptionally high in all Japanese populations eating fish and seafood daily. With obviously less CHD mortality rates, Japanese data were excluded from the following analyses on the association of CVD risks with $24 \mathrm{U}-\mathrm{T}$ or $-\mathrm{M}$, and 3960 individuals from $41 \mathrm{WHO}-$ CARDIAC Study populations were used for the following analyses [14].

\section{3-2) Influence of $T, M$ and combined $T$ and $M$ on CVD risks}

The mean of the individual data of $24 \mathrm{U}-\mathrm{T} / \mathrm{Cr}$ in 41 populations was 639.4 . Individuals with $24 \mathrm{U}-\mathrm{T} / \mathrm{Cr}$ equal to or above and below the mean are defined as high $\mathrm{T}$ and low $\mathrm{T}$ eaters. High $\mathrm{T}$ eaters were proven to have 


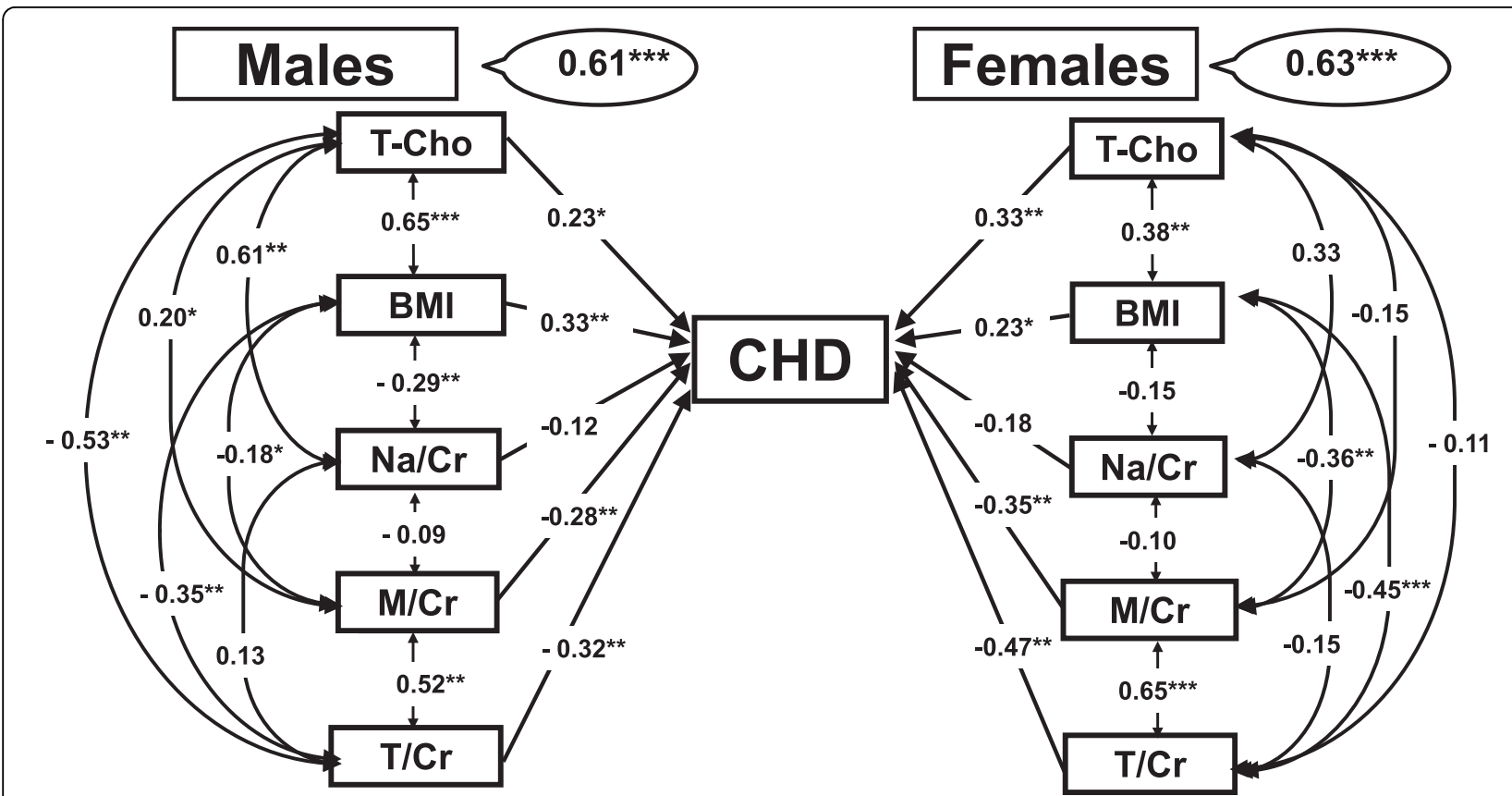

Figure 2 CARDIAC Study results of structural equation modeling of coronary heart diseases (CHD). Hypothetical pathway of 5 dietrelated factors in relation to CHD. T-Cho: total cholesterol, BMI: body mass index (body weight (kg)/height (m) $\left.{ }^{2}\right), \mathrm{Na} / \mathrm{Cr}$ : 24-hour urinary (24U) sodium ( $\mathrm{Na}, \mathrm{g})$ to creatinine $(\mathrm{Cr}, \mathrm{g})$ ratio, $\mathrm{M} / \mathrm{Cr}=24 \mathrm{U}$-magnesium $(\mathrm{M}, \mathrm{mg})$ to $\mathrm{Cr}$ ratio, $\mathrm{T} / \mathrm{Cr}=24 \mathrm{U}$-taurine $(\mathrm{T}$, mmol) to $\mathrm{Cr}$ ratio. Significant difference: ${ }^{*} p<0.05,{ }^{* *} p<0.01,{ }^{* * *} p<0.001$.

significantly less CVD risks such as obesity (body mass index, BMI), both SBP and DBP, T-Cho and atherogenic index (AI: T-Cho/HDL), an indicator of the risk for developing atherosclerosis and $\mathrm{CHD}$, than low $\mathrm{T}$ eaters [14]. Similarly, individuals with $24 \mathrm{U}-\mathrm{M} / \mathrm{Cr}$ equal to or above the mean 82.8 were proven to have significantly less CVD risks than those with $24 \mathrm{U}-\mathrm{M} / \mathrm{Cr}$ below the mean [14]. Therefore, we further analyzed these CVD risks in the individuals with both higher $24 \mathrm{U}-\mathrm{T} / \mathrm{Cr}$ and $-\mathrm{M} / \mathrm{Cr}$ ( $\geqq$ mean) (high T-M excreters) and proved that they had highly significantly lower BMI, SBP, DBP, TC and $\mathrm{AI}$ than low T-M excreters (< mean) as shown in Figure 3.

Since the differences in CVD risks between high T-M excreters and low excreters were statistically so high, we further analyzed the additional adverse effect of high salt $(\mathrm{Na})$ intake on low T-M excreters or the additional beneficial effect of low $\mathrm{Na}$ intake on high T-M excreters (Figure 4). High and low daily $\mathrm{T}-\mathrm{M}$ excreters were divided respectively into high and low excreters by the average of daily salt intake (10.2 g/day) estimated from 24U-Na. Differences in BMI, SBP, DBP and AI, except for T-Cho, remained statistically significant between high $\mathrm{T}-\mathrm{M}$ with low $\mathrm{Na}$ excreters and low $\mathrm{T}-\mathrm{M}$ with high $\mathrm{Na}$ excreters (Figure 4). In contrast to $\mathrm{Na}$ intake, $\mathrm{K}$ is regarded to beneficially affect CVD risks, particularly BP. Therefore, high and low T-M excreters were divided into high and low $\mathrm{K}$ excreters by the average of $\mathrm{K} / \mathrm{Cr}$ ratio, 46.1 (Figure 5). Differences in CVD risks except for $\mathrm{T}$-Cho remained statistically significant between high $\mathrm{K}$, high T-M excreters and low $\mathrm{K}$, low T-M excreters. High $\mathrm{K}$ and high $\mathrm{T}-\mathrm{M}$ excreters showed significantly higher $\mathrm{T}$-Cho than low $\mathrm{K}$ and low $\mathrm{T}$ - $\mathrm{M}$ excreters, indicating dietary habits to take more $\mathrm{K}$ linked to the diet to increase T-Cho higher. Therefore, we can conclude all these CVD risks (BMI, SBP, DBP, T-Cho, AI) are influenced more by differences in high and low $\mathrm{T}-\mathrm{M}$ excretion than differences in $\mathrm{Na}$ or $\mathrm{K}$ excretion regarded generally as adverse or beneficial dietary factors.

\section{4) Taurine and salt-related hypertension}

The population averages of both SBP and DBP in CARDIAC Study showed significantly positive association with the averages of $24 \mathrm{U}-\mathrm{Na}$ excretion in males but not in females in general. The association was significant only in SBP of postmenopausal women, indicating gender difference in salt-sensitive BP rise [39]. Since gene-environmental interactions such as nutrition was supposed to be involved in the salt sensitively, individual participants in the CARDIAC health survey were divided into greater or lesser $\mathrm{Na}$ excreters by the mean (10.2 g) of daily salt intake estimated from $24 \mathrm{U}-\mathrm{Na}$ excretion. As shown in Figure 6, greater $\mathrm{Na}$ excreters ( $\geqq$ mean) showed significantly higher SBP in total and in males 


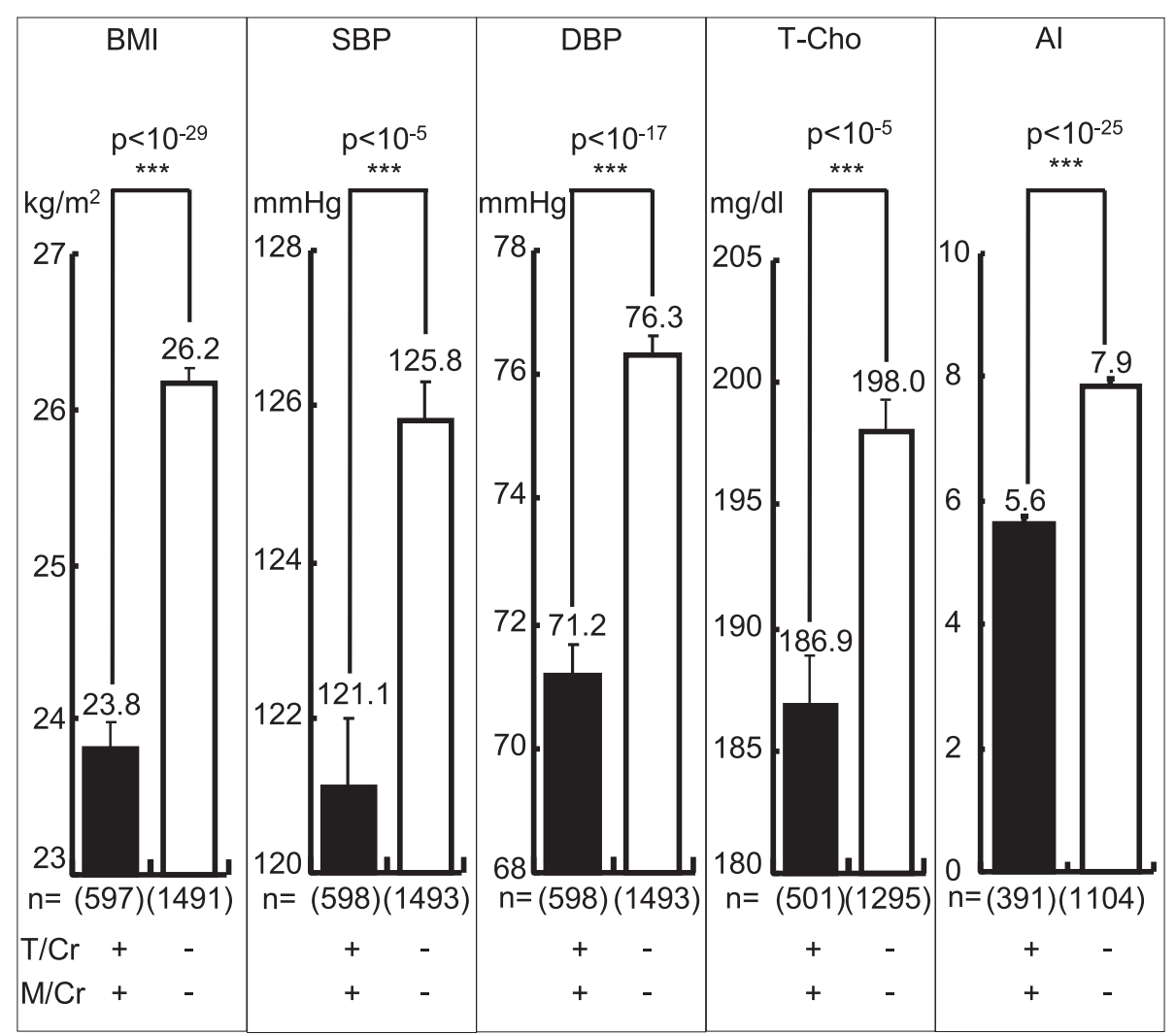

Figure 3 Cardiovascular risks in higher taurine-magnesium (T-M) excreters and lower T-M excreters. T/Cr $=24$-hour urinary (24U) taurine $(\mathrm{T}, \mathrm{mmol})$ to creatinine $(\mathrm{Cr}, \mathrm{g})$ ratio, $\mathrm{M} / \mathrm{Cr}=24 \mathrm{U}$-magnesium $(\mathrm{M}, \mathrm{mg})$ to $\mathrm{Cr}$ ratio. $\mathrm{T} / \mathrm{Cr}$ : $+\geqq$ mean $(=639.4),-<$ mean, $\mathrm{M} / \mathrm{Cr}$ : $+\geqq$ mean $(=82.8),-$ $<$ mean. BMI: body mass index (body weight $(\mathrm{kg}) /$ height $\left.(\mathrm{m})^{2}\right)$, SBP: systolic blood pressure, DBP: diastolic blood pressure, T-Cho: total cholesterol, Al: atherogenic index (T-Cho/high density lipoprotein cholesterol). Parenthesis indicates a number of participants. Significant difference: ${ }^{* * *} p<0.001$.

and females, respectively. However, in DBP the differences in total and males were significant, but there was no significant difference in females indicating the consistency of the individual data analyses with the population average data.

Heart rate (HR) is related to CVD risk in CARDIAC data [40] as well as in other epidemiological data indicating lower HR is significantly related to longer lifeexpectancy in the elderly $[41,42]$. Since CARDIAC Study analysis on the population averages showed that higher $24 \mathrm{U}-\mathrm{T}$ was significantly associated with lower BMI, SBP, DBP and HR, the involvement of $\mathrm{T}$ in salt-sensitive BP rise was analyzed by dividing greater $\mathrm{Na}$ excreters in males into those with higher HR ( $\geqq$ mean, 71.5 beat/ min) and with slower HR (< mean). As shown in Figure 7 , greater Na excreters with higher HR had significantly higher SBP and DBP than those with lower HR, indicating higher HR with higher salt intake estimated by greater 24U-Na excretion was related with salt-sensitive $\mathrm{BP}$ rise in which sympathetic neural component was supposedly involved. Therefore, greater $24 \mathrm{U}-\mathrm{Na}$ excreters with higher and lower HR were both divided into high and low $\mathrm{T}$ excreters $(\mathrm{T} / \mathrm{Cr} \geqq$ or $<$ mean). Higher T excreters' SBP and DBP were significantly lower only in greater $\mathrm{Na}$ excreters with high $\mathrm{HR}$, and there was no significant difference in both SBP and DBP of greater $\mathrm{Na}$ excreters with lower HR. These findings are suggestive of T's BP reducing effect on neural regulation of BP rise induced by salt loading as in experimental studies in rat models of salt-sensitive hypertension, SHRSP and DOCA salt hypertensive rats. $\mathrm{T}$ administration decreased BP more markedly in saltsensitive SHRSP than in SHR and in normotensive Wister-Kyoto rats [9], and in salt-loaded DOCA hypertensive rats in which $\mathrm{T}$ reduced $\mathrm{BP}$ increase as well as catecholamine excretion [43]. The latter indicates the neurogenic development of salt-induced hypertension. We previously reported that BP and catecholamine responses to cold exposure stress in young volunteers were significantly greater in those with family history of hypertension than the other without the family history $[15,44]$. Therefore, our present epidemiological data 


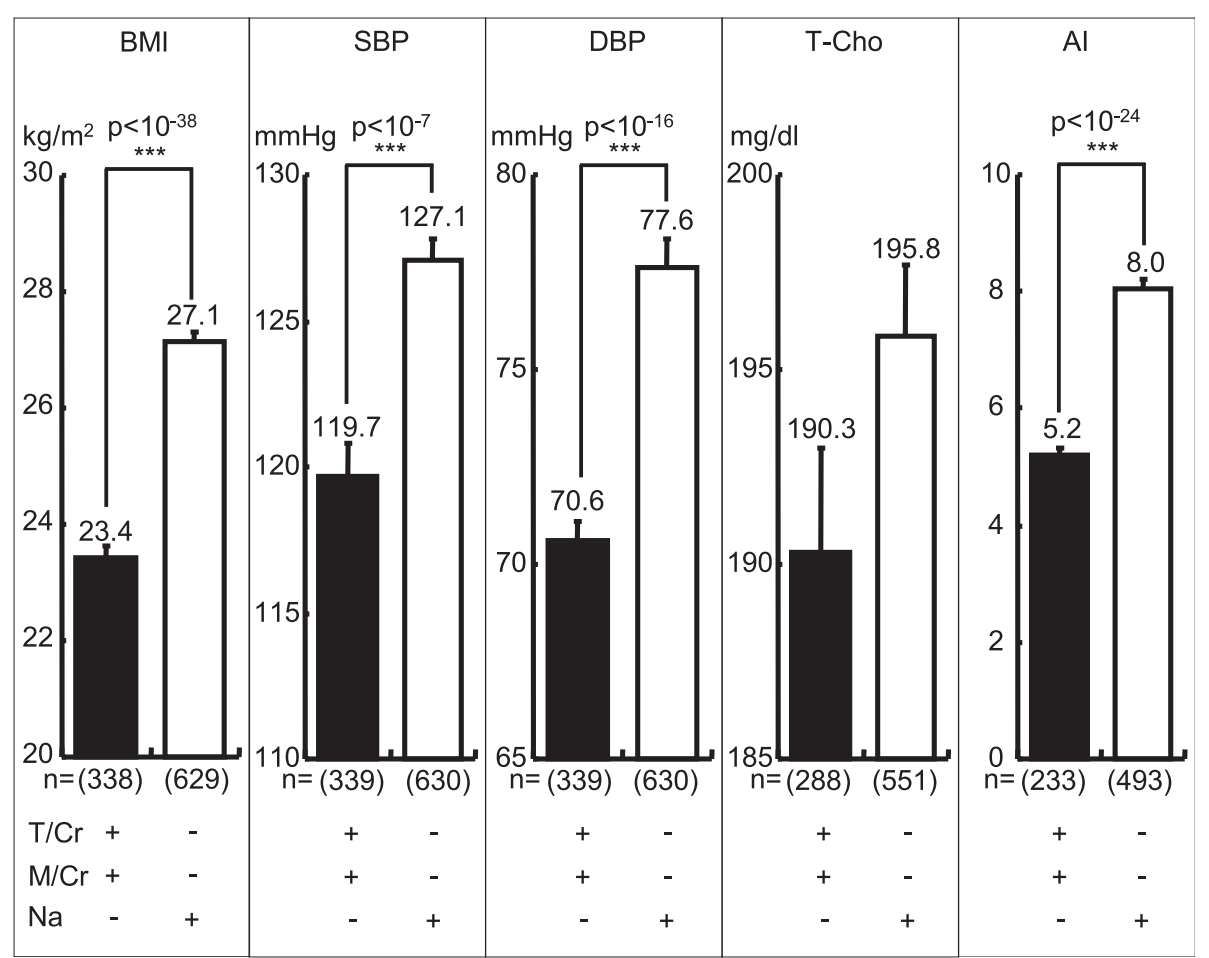

Figure 4 Cardiovascular disease risks in higher and lower taurine-magnesium excreters with greater or lesser sodium excretion. $\mathrm{T} / \mathrm{Cr}=$ 24-hour urinary (24U) taurine $(\mathrm{T}, \mathrm{mmol})$ to creatinine $(\mathrm{Cr}, \mathrm{g})$ ratio, $\mathrm{M} / \mathrm{Cr}=24 \mathrm{U}$-magnesium ( $\mathrm{M}, \mathrm{mg})$ to $\mathrm{Cr}$ ratio, $\mathrm{Na}$ : $24 \mathrm{U}$-sodium ( $\mathrm{Na}$ as $\mathrm{NaCl}, \mathrm{g} /$ day). T/Cr: $+\geqq$ mean $(=639.4),-<$ mean, M/Cr: $+\geqq$ mean $(=82.8),-<$ mean, Na: $+\geqq$ mean $(=10.14),-<$ mean. BMl: body mass index (body weight $(\mathrm{kg}) /$ height $\left.(\mathrm{m})^{2}\right)$, SBP: systolic blood pressure, DBP: diastolic blood pressure, T-Cho: total cholesterol, Al: atherogenic index (T-Cho/high density lipoprotein cholesterol). Parenthesis indicates a number of participants. Significant difference: ${ }^{* * *} p<0.001$.

indicating that among greater $\mathrm{Na}$ excreters with greater HR, higher $\mathrm{T}$ excreters showed lower BP than lower $\mathrm{T}$ excreters support the possible neurogenic component modulated by $\mathrm{T}$ in the salt sensitive hypertension.

\section{5) Taurine (T) and magnesium (M) in high and low risk populations}

Since the individuals with greater $\mathrm{T}$ and $\mathrm{M}$ intakes estimated from $24 \mathrm{U}$ excretions had significantly lower CVD risks than lesser $\mathrm{T}$ and $\mathrm{M}$ excreters [14], typical populations, in which the population averages of both $24 \mathrm{U}-\mathrm{T}$ and $-\mathrm{M}$ were higher and lower than the average of CARDIAC population samples, were investigated for their life style and dietary habits for comparison. Among 41 WHO-CARDIAC populations, Guiyang people in south-west China near Guangzhou showed both higher $24 \mathrm{U}-\mathrm{T}$ and $-\mathrm{M}$ than the averages among total CARDIAC populations (Figure 8) [38]. In contrast, St. John's population living on Newfoundland Island, Canada had both lower $\mathrm{T}$ and $\mathrm{M}$ than the averages of the CARDIAC populations. In Guiyang we first carried out a health survey in 1988 and repeated the survey in 1997 after the rapid economic development in China [38]. There lived Miao, Pui and other minority people who were still keeping a traditional dietary habit, such as eating daily soy beans and various soy products, baked bean curd (coagulated soy protein) prepared by M-rich water and eating much fresh water fish, indicating that their rich sources of $\mathrm{T}$ and $\mathrm{M}$ are fresh water fish, soy and soy products. The prevalence of CVD risks was remarkably low in Guiyang, and they had almost no risks of obesity, hypertension and hypercholesterolemia indicating that their dietary habit of consuming high levels of both $\mathrm{T}$ and $\mathrm{M}$ is ideal for CVD prevention (Figure 9). Correspondingly, the Guiyang population had significantly lower BMI, both SBP and DBP, T-Cho level and $\mathrm{AI}$ than the averages of CARDIAC populations taking various amounts of $\mathrm{T}$ and $\mathrm{M}$ in other parts of the world. In contrast, Newfoundlanders in St. John's were the descendants of Scottish and Irish immigrants and were engaged in farming sheep on the island and also in fishing for abundant sea water fish near the island. They traditionally salted fresh fish in barrels for shipping them as preserved food to Canada and US, and did not eat raw fish like the Japanese. They were eating mainly meat products so that their $\mathrm{T}$ and $\mathrm{M}$ excretions were lower than the averages of CARDIAC populations. CVD risks of Newfoundlanders both in male and females 


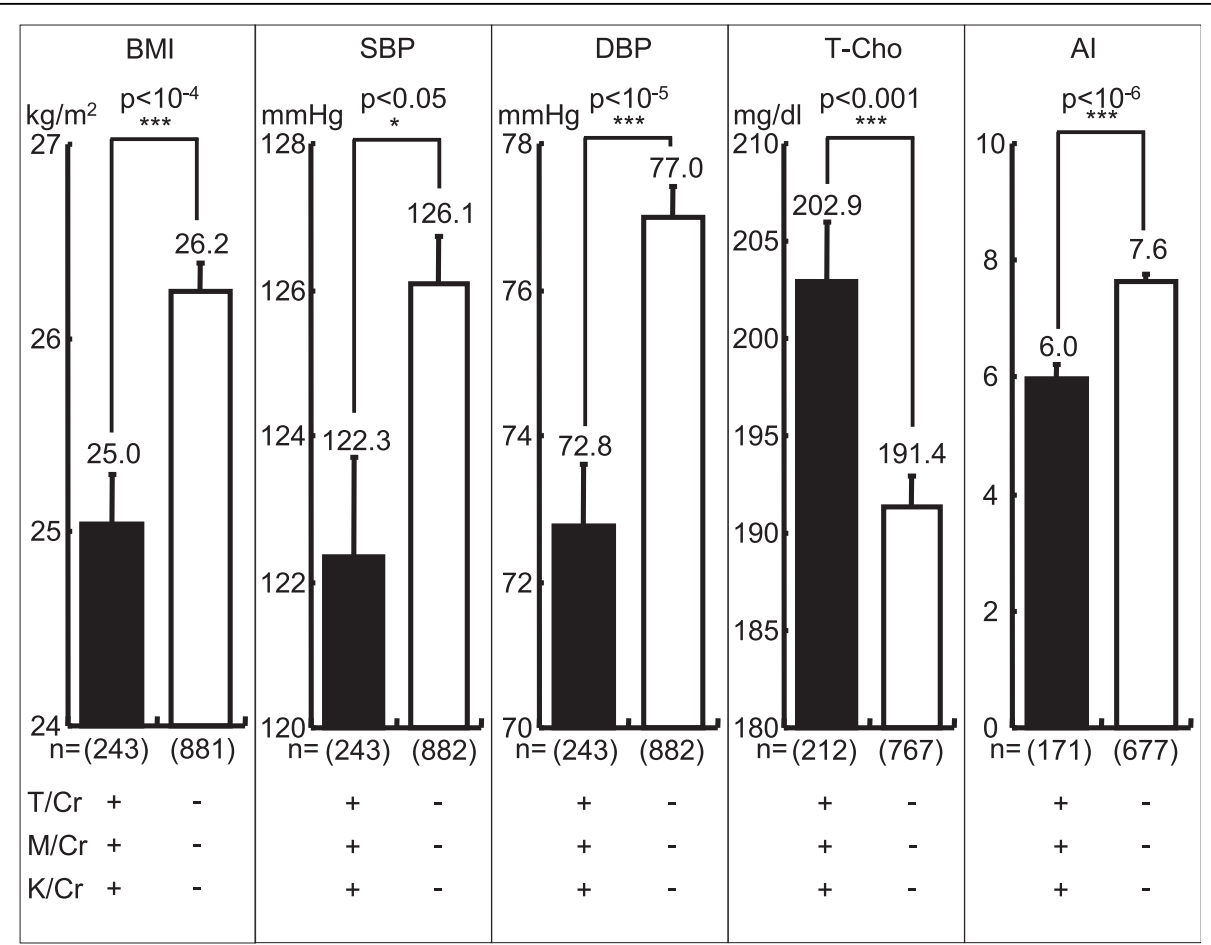

Figure 5 Cardiovascular disease risks in higher taurine-magnesium-potassium (T-M-K) excreters and lower T-M-K excreters. T/Cr: 24hour urinary $(24 \mathrm{U})$ taurine $(\mathrm{T}, \mathrm{mmol})$ to creatinine $(\mathrm{Cr}, \mathrm{g})$ ratio, $\mathrm{M} / \mathrm{Cr}$ : $24 \mathrm{U}$-magnesium $(\mathrm{M}, \mathrm{mg})$ to $\mathrm{Cr}$ ratio, $\mathrm{K} / \mathrm{Cr}$ : 24U-potassium (K, milliequivalent) to $\mathrm{Cr}$ ratio. $\mathrm{T} / \mathrm{Cr}$ : $+\geqq$ mean $(=639.4),-<$ mean, $\mathrm{M} / \mathrm{Cr}$ : $+\geqq$ mean $(=82.8),-<$ mean, $\mathrm{K} / \mathrm{Cr}$ : $+\geqq$ mean $(=46.1),-<$ mean. BMI: body mass index (body weight $(\mathrm{kg}) /$ height $(\mathrm{m})^{2}$ ), SBP: systolic blood pressure, DBP: diastolic blood pressure, T-Cho: Total Cholesterol, Al: Atherogenic index (Total cholesterol/HDL cholesterol). Parenthesis indicates a number of participants. Significant difference: ${ }^{*} p<0.05,{ }^{* * *} p<0.001$.

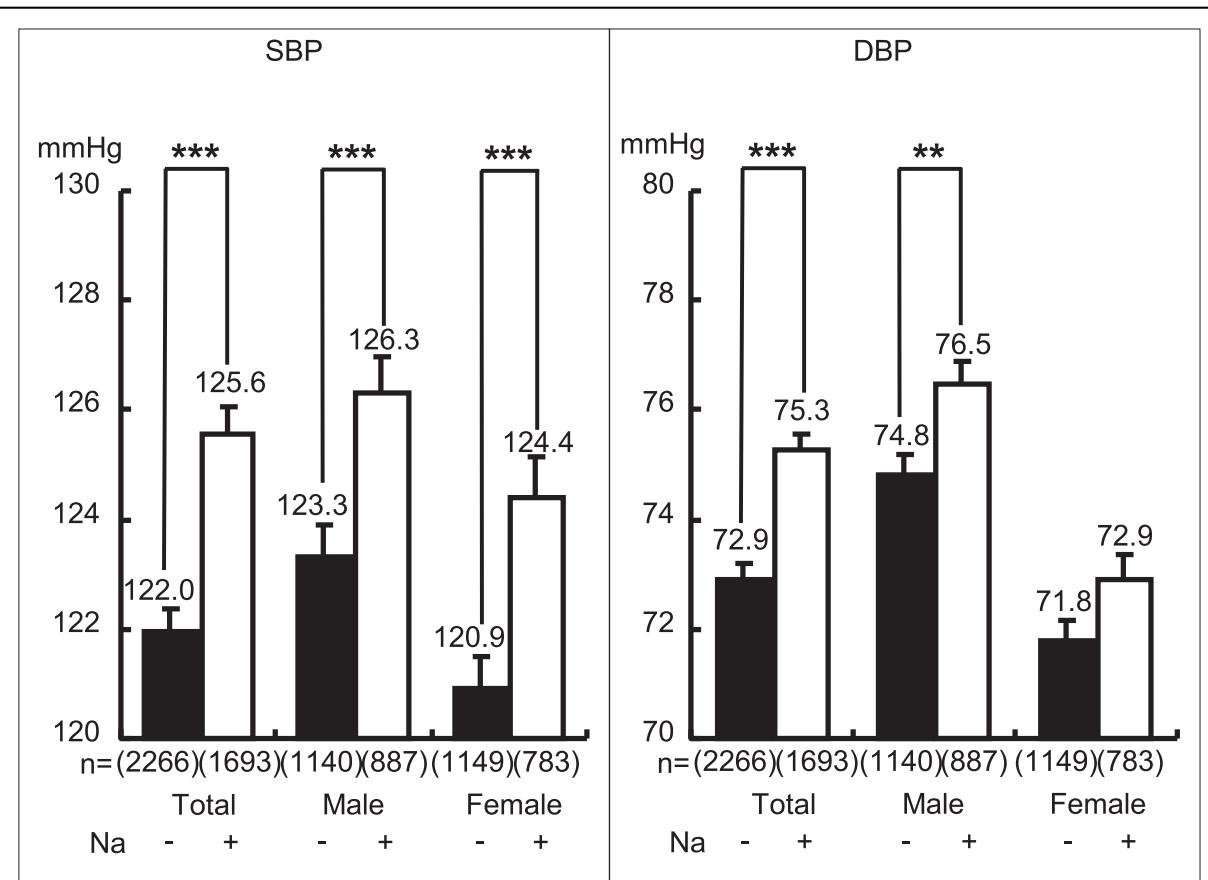

Figure 6 Blood pressure in greater or lesser 24-hour urinary sodium. Na: 24-hour urinary (24U) sodium ( $\mathrm{Na}$ as $\mathrm{NaCl}, \mathrm{g} / \mathrm{day}), \mathrm{Na}$ : + $\geqq$ mean (= 10.14), - < mean. SBP: systolic blood pressure, DBP: diastolic blood pressure. Parenthesis indicates a number of participants. Significant difference: ${ }^{* *} p<0.01,{ }^{* * *} p<0.001$. 

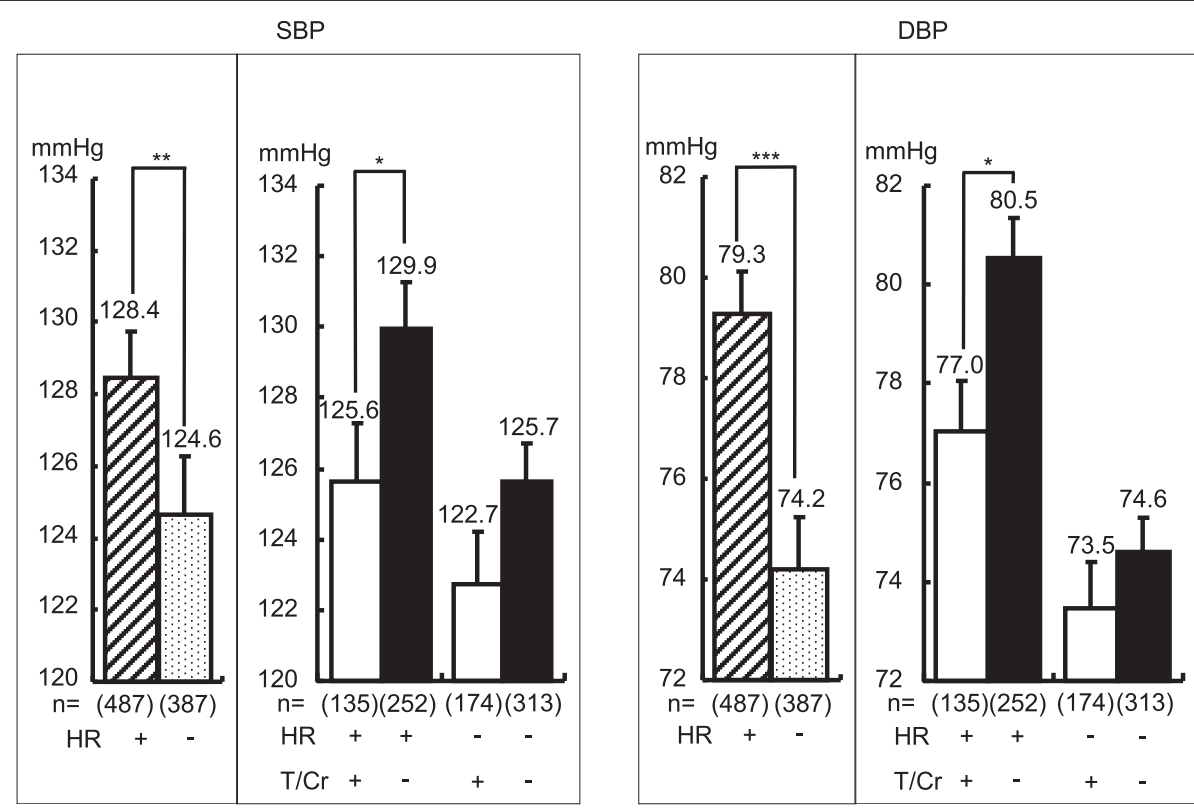

Figure 7 Effect of taurine on blood pressure in male greater salt eaters with faster heart rate. Male greater salt eaters were defined as shown in Figure 6 and analyzed only in those whose heart rate (HR) were recorded. T/Cr: 24-hour urinary taurine $(T$, mmol) to $\mathrm{creatinine}(\mathrm{Cr}, \mathrm{g})$ ratio. T/Cr: $+\geqq$ mean $(=639.4),-<$ mean, HR: $+\geqq$ mean $(=72.9),-<$ mean. SBP: systolic blood pressure, DBP: diastolic blood pressure

Parenthesis indicates a number of participants. Significant difference: ${ }^{*} p<0.05,{ }^{* *} p<0.01,{ }^{* * *} p<0.001$.

were significantly higher than those of the Guiyang people who were consuming high levels of both $\mathrm{T}$ and $\mathrm{M}$ (Figure 9). St. John's population was known for their high CVD mortality rates, whereas Guiyang's population was known for their low CVD mortality rates and their longevity in China. These data based on the individual biomarkers related to $\mathrm{T}$ and $\mathrm{M}$ intakes indicate that eating foods containing both $\mathrm{T}$ and $\mathrm{M}$ is good for the physiological regulation of the homeostasis of the cells and human bodies. This dietary habit may contribute to the prevention of CVD and thus to the healthy longevity of humans. CVD risks are definitely lower in individuals excreting high levels of $24 \mathrm{U}-\mathrm{T}$ and $-\mathrm{M}$. Among CARDIAC populations, Guiyang population excreting and supposedly taking both had definitely less CVD risks than the St. John's population who were not excreting and taking both elements. Therefore, seafood, soy, nuts, and milk taken regularly as the sources of $\mathrm{T}$ and $\mathrm{M}$ could be recommended for CVD prevention.

\section{6) Bush food rich in $T$ and $M$, needed for health}

We are still continuing our CARDIAC health examination since we started the world wide study in 1985 in coordination with WHO. Our recent study on Australian Aboriginal people for over past 7 years demonstrated that they are presently suffering most severely from lifestyle-related diseases in comparison with the other CARDIAC populations $[38,45]$. Obesity, hypertension and diabetes in Aboriginals start 20 to 30 years younger than other Australian and Japanese people (Figure 10) [45]. When they lost bush food, their M intake was greatly decreased because bush food consisting of nuts, seeds, grains, beans, fruits and seaweeds contains much $M$ (Figure 11). Aboriginals living near the coast of the state of Victoria used to utilize various foods from the sea and fresh water. Aboriginals living in Framlingham, Victoria were known to start the culture of eels 8,000 years ago and there were several large shell mounds, the piling up of various shells like mountains at the sea side not far from Melbourne, indicating that Aboriginals' life had been highly dependent on their bush food as well as on the seafood, both the richest sources of $\mathrm{T}$ and $\mathrm{M}$. Before the colonials were established, their daily foods were clams, various shells and fish containing a large amount of $\mathrm{T}$ (Figure 12), and also their traditional smoked eels, which were a rich source of $\mathrm{T}$ and DHA and eaten as their common preserved food. As hunters and food gatherers, Aboriginals, according to the 2-week food intake analysis report, were supposed to live by taking their energy, for example, $34 \%$ from complex carbohydrates, $13 \%$ from fat and $53 \%$ from protein [46]. Although we do not know exactly what they ate, we tentatively suppose, for example, they ate $400 \mathrm{~g}$ of yam, $300 \mathrm{~g}$ of frogs, $5 \mathrm{~g}$ of almonds, $140 \mathrm{~g}$ of bream, $200 \mathrm{~g}$ of clams, $300 \mathrm{~g}$ of snails. These foods contain about $100 \mathrm{~g}$ of carbohydrates, $15 \mathrm{~g}$ of fat and $170 \mathrm{~g}$ of protein and correspond roughly to $1300 \mathrm{kcal} /$ day. The daily intake of $\mathrm{T}$ and $\mathrm{M}$ from these 

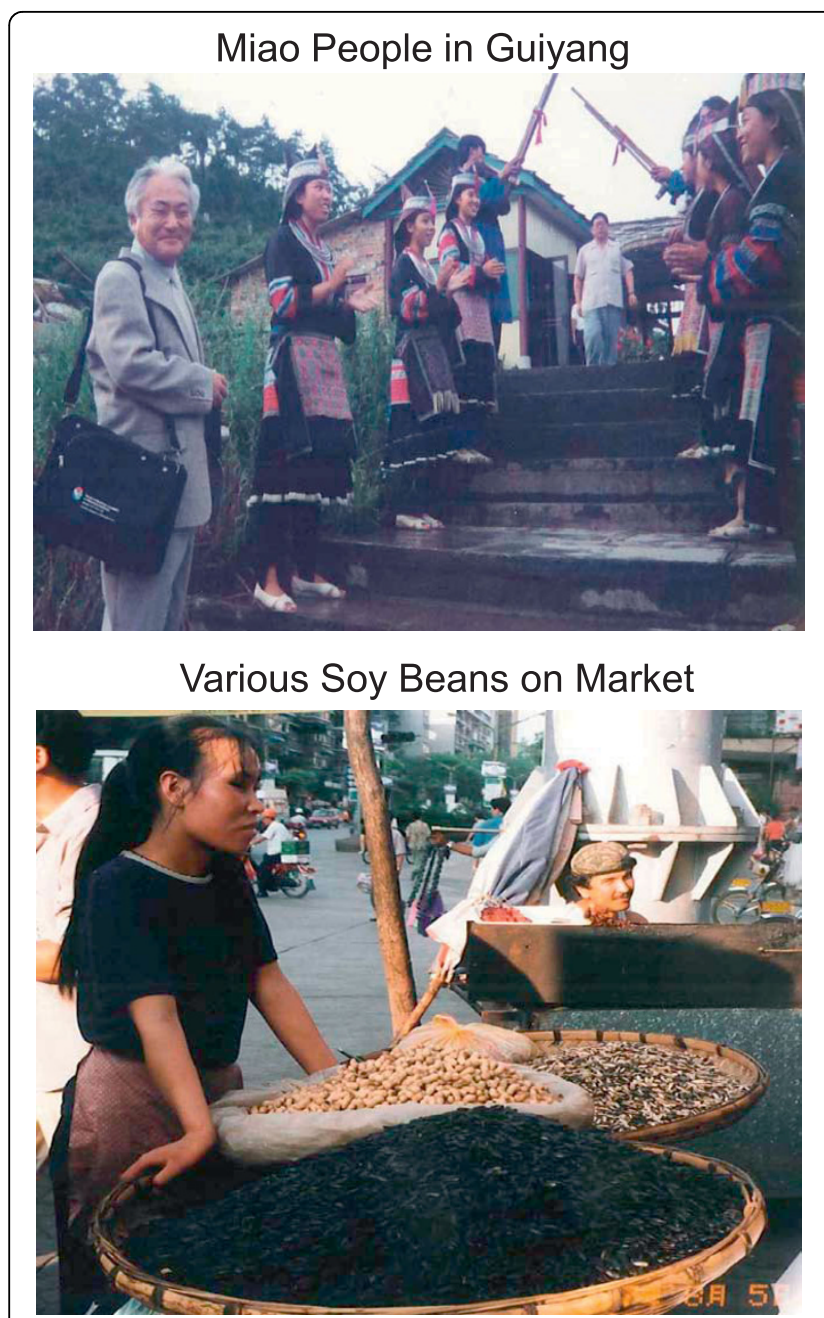
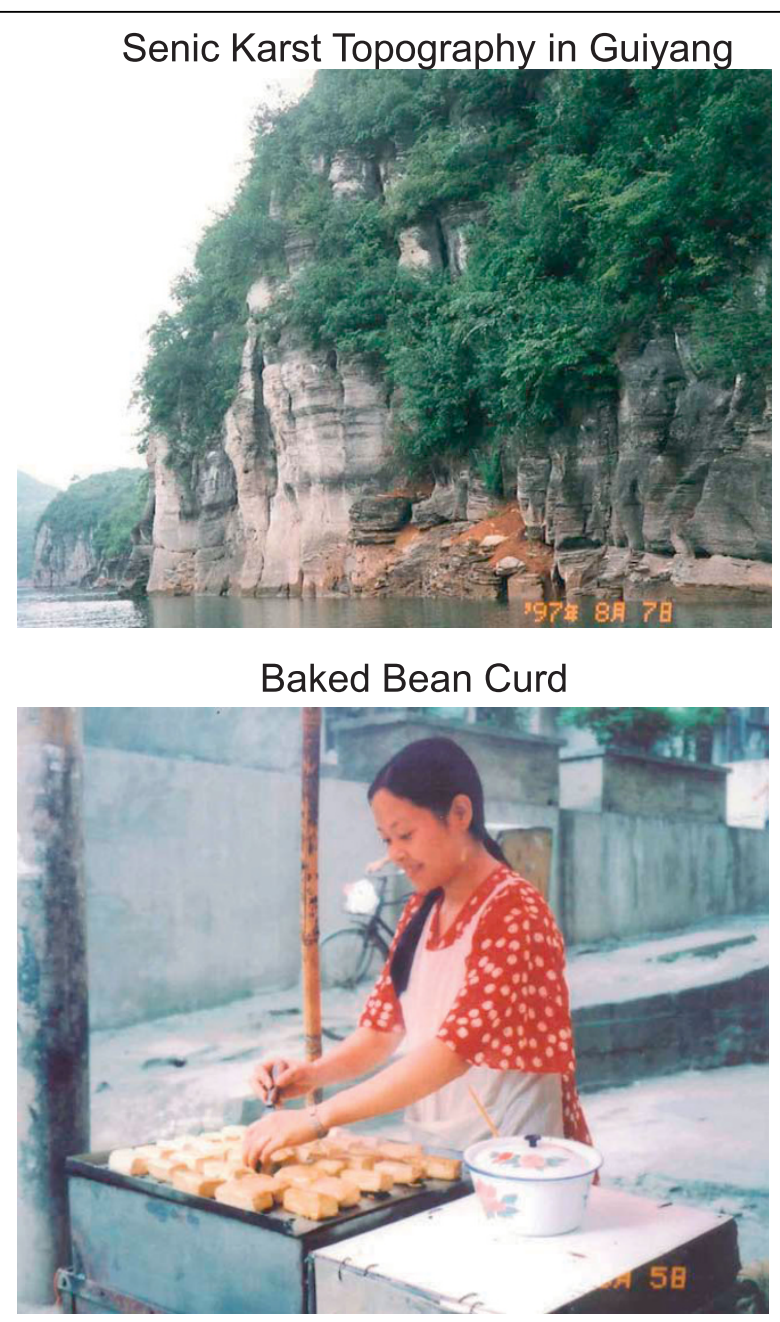

Figure 8 Food culture, lifestyle and topographic background of Guiyang people in China.

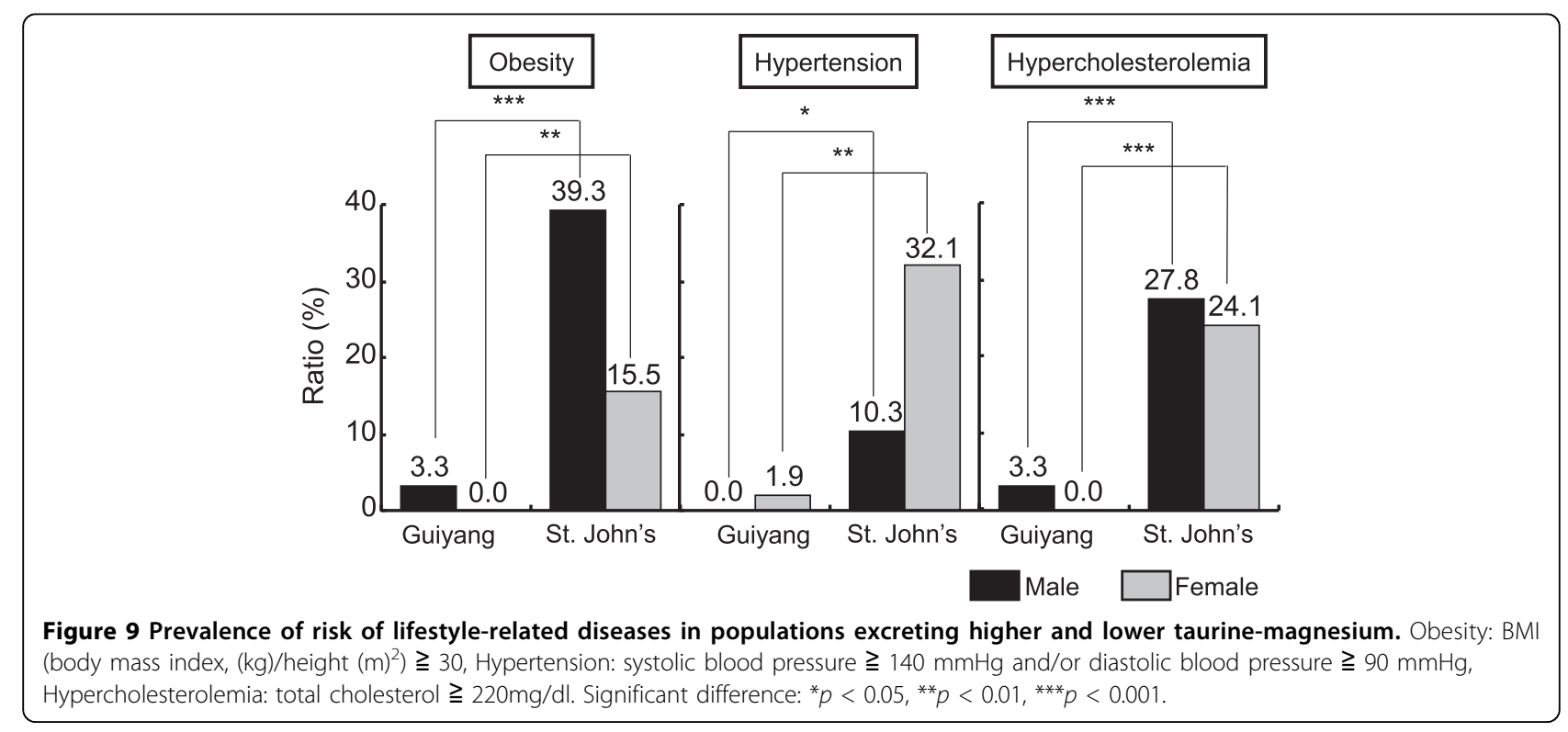




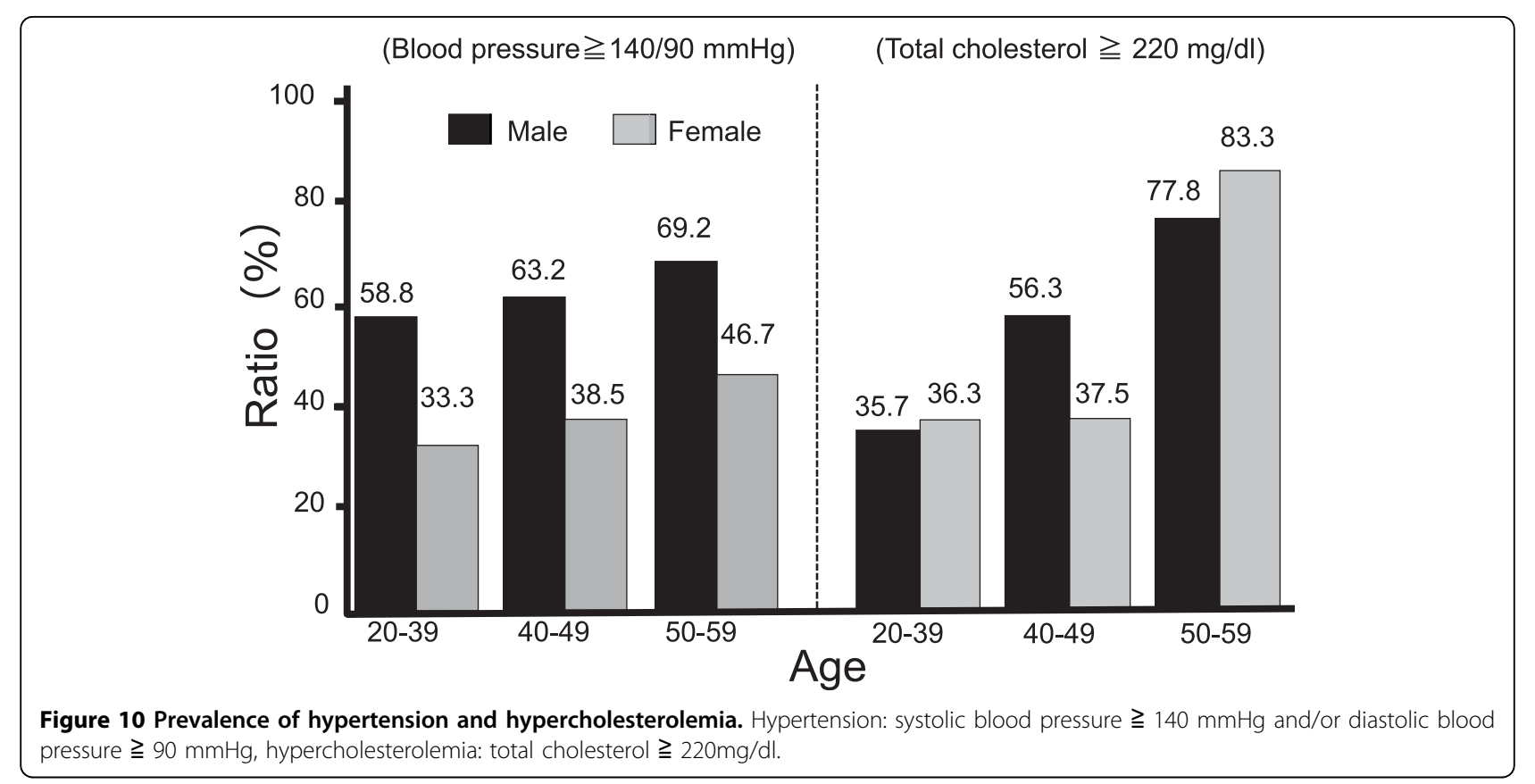

foods is about $3200 \mathrm{mg}$ and $640 \mathrm{mg}$, respectively, both being far higher than the average of CARDIAC populations in the world.

Moreover, they had no salt at all before Captain Cock reached Australia at the beginning of 18th century. Our CARDIAC data help us to speculate how Aboriginals should have been free from CVD risks, based on our comparison of CARDIAC Study individuals consuming a high amount of $\mathrm{T}$ and $\mathrm{M}$ but less $\mathrm{Na}$ with those consuming less $\mathrm{T}$ and $\mathrm{M}$ but a great amount of Na. CVD risks were clearly and highly significantly lower in the CARDIAC Study participants taking a high amount of $\mathrm{T}$ and $\mathrm{M}$ but less $\mathrm{Na}$, corresponding to the nutritional balance of Aboriginal ancestors eating traditional bush food. High $\mathrm{T}$ and $\mathrm{M}$ but less Na-containing foods were supposed to be taken commonly by the ancestors of humans living on hunter-gatherers' foods like Aboriginal ancestors for nearly 10 times longer than the period after the start of agriculture and farming. Therefore, these dietary

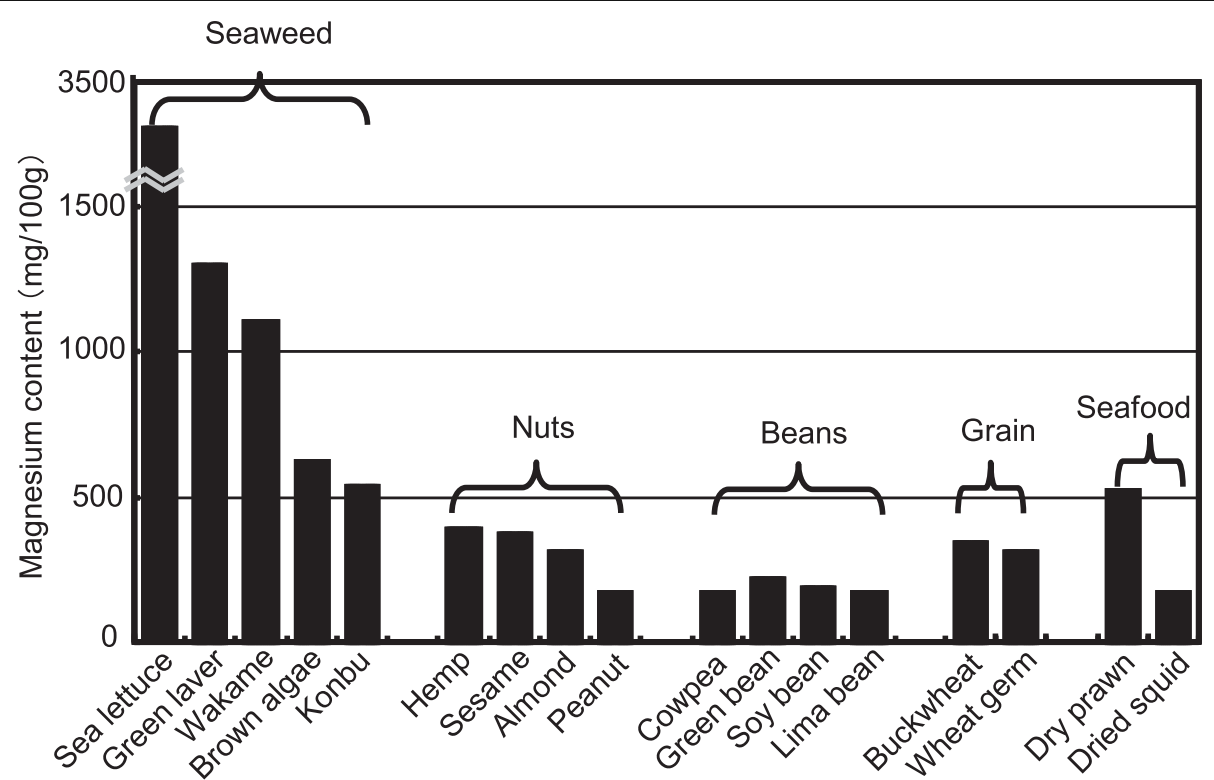

Figure 11 Magnesium contents of typical dry food. 


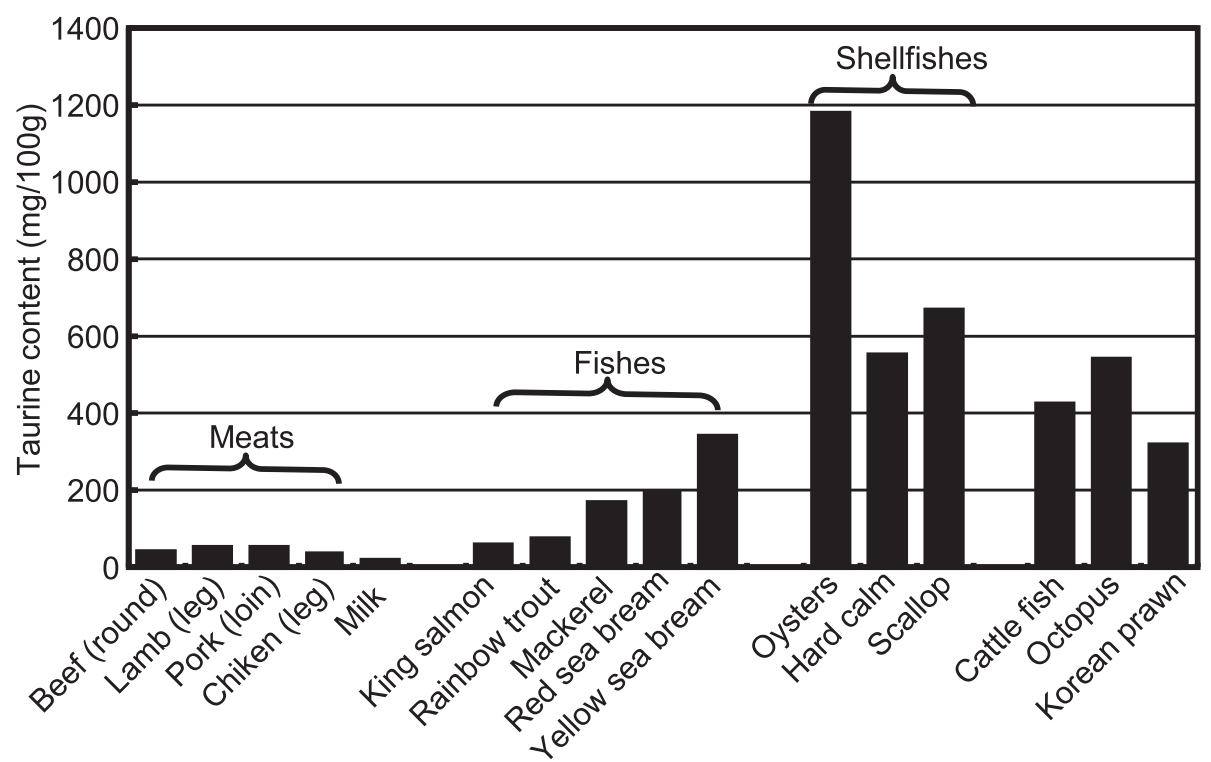

Figure 12 Taurine contents of typical food.

conditions seem to be more suitable for the human genome to live a healthy life without CVD.

\section{7) $T$ and $M$ in cardiovascular pathophysiology and longevity}

Both $\mathrm{T}$ and $\mathrm{M}$ are basically important for the maintenance of life. $\mathrm{T}$ is involved in cellular physiology by its effect on osmoregulation, anti-oxidant, membrane stabilization and calcium regulation [47-49], and also on lipid metabolism related to dyslipidemia and atherosclerosis through its role in bile acid conjugation $[47,48]$. Recently, T-conjugated endogenous bile acid derivative, ursodeoxycholic acid was focused on as a chemical chaperone which was proven to reduce endoplasmic reticulum (ER) stress and restore glucose homeostasis in a mouse model of type 2 diabetes [50]. The alleviation of ER-stress may restore insulin sensitivity in the liver, muscle and adipose tissue, thus contributing to the resolution of fatty liver diseases, diabetes and obesity. High dietary $\mathrm{T}$ administration was proven to reduce apoptosis and atherosclerosis possibly via normalization of ER stress [51].

Moreover, in relation to the ageing of vascular tissue and the accelerated senescence of endothelial progenitor cell (EPC) noted in SHRSP [52], $\mathrm{T}$ was reported to attenuate EPC senescence in SHRSP and to modulate clinically the deterioration of endothelial function in smokers [53-55]. These classical and new concepts of the pathophysiological roles of $\mathrm{T}$ suggest that a high $\mathrm{T}$ intake may contribute to longevity through CVD health and lifestyle-related diseases.
On the other hand, $\mathrm{M}$ is the 8th abundant element in the weight, the number of atoms and the volume percentage of all atoms on the earth. It is also the most abundant intracellular divalent cation and is involved in the various biological functions of about 300 enzymes as their coenzyme. They include all enzymatic reactions requiring $\mathrm{ATP}$, such as $\mathrm{Na}-\mathrm{K}$ ATPase, important for intracellular ionic balance. Therefore, $M$ is supposed to be causatively and clinically related to cardiovascular health, hypertension and diabetes [56-58]. Lowering of intracellular free $M$ was observed in SHRSP in the process of aging and development of hypertension [59]. Dietary M supplementation increased intralymphocytic free $M$ and attenuated the grade of hypertension [60]. These experimental findings were recently confirmed clinically in patients with mild hypertension whose ambulatory BP was significantly decreased concomitantly with the increased intracellular free $\mathrm{M}$ and $\mathrm{K}$, and decreased intracellular free $\mathrm{Ca}$ and $\mathrm{Na}$, by the dietary supplementation of $600 \mathrm{mg}$ of pidolate $M$ [61]. In this clinical experiment, $M$ supplementation increased serum $\mathrm{M}$ and $24 \mathrm{U}$ excretion of $\mathrm{M}$. Therefore, the aforementioned epidemiologically observed $24 \mathrm{U}-\mathrm{M}$ excretion was regarded to correspond to dietary $M$ intake. $M$ was also reported to decrease BP by inhibiting sympathetic nerve by blocking $\mathrm{N}$-type Ca channels [62].

Since the evolutional origin of life of human beings was inside the sea containing abundant $M$ and food gatherers lived on seafood rich in $\mathrm{T}$, both $\mathrm{M}$ and $\mathrm{T}$ are assumed to be essential for the homeostasis maintaining cardiovascular health. 


\section{Conclusions}

In addition to the pathophysiological and various physiological functions of $\mathrm{T}$, the preventive effect of $\mathrm{T}$ was first experimentally demonstrated in hypertensive rat models, SHR and SHRSP developing hypertension and stroke genetically. World-wide epidemiological studies conducted for the last 25 years revealed that $24 \mathrm{U}-\mathrm{T}$ was inversely associated with the age-adjusted mortality rates of stroke and CHD. High 24U-T, particularly combined with high $\mathrm{M}$ excretion, was associated highly significantly with lower CVD risks, obesity, hypertension, hypercholesterolemia and AI. These findings indicate the consistency of the beneficial $\mathrm{T}$ effects on animal models and humans proven either experimentally or epidemiologically. Such consistency of the extensive basic and epidemiological findings of $\mathrm{T}$ effects and the effects combined with $M$ indicates greater prospective for $\mathrm{T}$ to contribute to the nutritional prevention of CVD and lifestyle-related diseases.

\section{List of abbreviations used}

Al: atherogenic index, BMI: body mass index, ALR: arterio-lipidosis prone rats, Ca: calcium, CARDIAC Study: Cardiovascular Diseases and Alimentary Comparison study, CHD: coronary heart diseases, Cr: creatinine, CRP: Creactive protein, CVD: cardiovascular diseases, DBP: diastolic blood pressure, ER: endoplasmic reticulum, HDL: high density lipoprotein, HR: heart rate, $\mathrm{K}$ : potassium, LDL: low density lipoprotein, M: magnesium, Na: sodium, NASH: nonalcoholic steato-hepatitis, SBP: systolic blood pressure, SHR: spontaneously hypertension rats, SHRSP: stroke-prone spontaneously hypertension rats, T: taurine, T-Cho: total cholesterol, 24U: 24-hour urine.

\section{Acknowledgements}

Epidemiological and experimental studies by the authors' group was supported partly by Grants-in-Aid for Scientific Research from the Ministry of Education, Culture, Sports, Science and Technology, the Japanese government, and by the donations to $\mathrm{WHO}$ from many companies and over 300,000 individuals in total. Our appreciation should be extended to all participants in WHO-CARDIAC Study and to collaborating workers in worldwidely distributed study centers.

This article has been published as part as part of Journal of Biomedical Science Volume 17 Supplement 1, 2010: Proceedings of the 17th International Meeting of Taurine. The full contents of the supplement are available online at http://www.jbiomedsci.com/supplements/17/S1.

\section{Authors' contributions}

YY designed taurine-related studies and prepared for this review. MM and HM conducted epidemiological surveys. TT contributed to the data analysis. AM conducted laboratory analyses. KK helped experimental studies and reference collection.

\section{Competing interests}

The authors declare that they have no competing interests.

Published: 24 August 2010

\section{References}

1. Okamoto K, Aoki K: Development of a strain of spontaneously hypertensive rats. Jpn Circ J 1963, 27:282-293.

2. Yamori Y: Development of the spontaneously hypertensive rat (SHR) and of various spontaneous rat models, and their implications. Handbook of Hypertension Elsevierde Jong W. Amsterdam 1984, 224-239.

3. Okamoto T, Yamori Y, Nagaoka A: Establishment of the stroke-prone spontaneously hypertensive rat (SHR). Circ Res 1974, 34/35:143-153.
4. Yamori Y: The stroke-prone spontaneously hypertensive rat: Contribution to risk factor analysis and prevention of hypertensive diseases. Handbook of Hypertension Elsevierde Jong W. Amsterdam 1984, 240-255.

5. WHO and WHO Collaborating Centers: CARDIAC (Cardiovascular Diseases and Alimentary Comparison) study protocol. Shimane/Geneva 1986.

6. Yamori Y: Hypertension and biological dietary markers in urine and blood: A progress report from the CARDIAC study group. New Horizons in Preventing Cardiovascular Diseases ElsevierYamori Y, Strasser T. Amsterdam 1989, 111-126.

7. Yamori Y, Nara Y, Mizushima S, Murakami S, Ikeda K, Sawamura M, Nabika T, Horie R: Gene-environment interaction in hypertension, stroke and atherosclerosis in experimental models and supportive findings from a world-wide cross-sectional epidemiological survey: a WHO-CARDIAC study. Clin Exp Pharmacol Physiol Suppl 1992, 20:43-52.

8. Yamori Y, Liu L, Mizushima S, Ikeda K, Nara Y: Male cardiovascular mortality and dietary markers in 25 population samples of 16 countries. $J$ Hypertens 2006, 24:1499-1505.

9. Nara $Y$, Yamori $Y$, Lovenberg W: Effect of dietary taurine on blood pressure in spontaneously hypertensive rats. Biochem Pharmacol 1978, 27:2689-2692.

10. Yamori $Y$, Nara $Y$, Ikeda K, Mizushima S: Is taurine a preventive nutritional factor of cardiovascular diseases or just a biological marker of nutrition? Adv Exp Med Biol 1996, 403:623-629.

11. Yamori Y, Liu L, Ikeda K, Miura A, Mizushima S, Miki T, Nara Y: Distribution of twenty-four hour urinary taurine excretion and association with ischemic heart disease mortality in 24 populations of 16 countries: results from the WHO-CARDIAC study. Hypertens Res 2001, 24:453-457.

12. Yamori Y, Liu L, Mori M, Sagara M, Murakami S, Nara Y, Mizushima S: Taurine as the nutritional factor for the longevity of the Japanese revealed by a world-wide epidemiological survey. Adv Exp Med Biol 2009, 643:13-25.

13. Chen SW, Chen YX, Shi J, Lin Y, Xie WF: The restorative effect of taurine on experimental nonalcoholic steatohepatitis. Dig Dis Sci 2006, 51:2225-2234.

14. Yamori Y, Taguchi T, Mori M, Mori H: Low cardiovascular risks in the middle aged males and females excreting greater 24-hour urinary taurine and magnesium in 41 WHO-CARDIAC study populations in the world. J Biomed Sci., Citation from within the same Supplement..

15. Yamori Y: Predictive and preventive pathology of cardiovascular diseases. Acta Pathol Jpn 1989, 39:683-705.

16. Yamori $Y$, Horie $R$, Nara $Y$, Tagami M, Kihara M, Mano M, Ishio H: Pathogenesis and dietary prevention of cerebrovascular diseases in animal models and epidemiological evidence for the applicability in man. Prevention of Cardiovascular Diseases, an Approach to Active Long Life ElsevierYamori Y, Lenfant C. Amsterdam 1987, 163-177.

17. Tagami M, Tsukada T, Kubota A, Nara A, Yamori Y: Immunocytochemical study of cerebral perforating arteries in patients with cerebral infactions. Acta Histochem Cytochem 1993, 26:109-115.

18. Schuller Levis GB, Park E: Taurine and its chloramine: modulators of immunity. Neurochem Res 2004, 29:117-126.

19. Sun Jang J, Piao S, Cha YN, Kim C: Taurine chloramine activates Nrf2, increases HO-1 expression and protects cells from death caused by hydrogen peroxide. J Clin Biochem Nutr 2009, 45:37-43.

20. Alexander RW: Theodore Cooper Memorial Lecture. Hypertension and the pathogenesis of atherosclerosis. Oxidative stress and the mediation of arterial inflammatory response: a new perspective. Hypertension 1995, 25:155-161.

21. Ridker PM, Rifai N, Rose L, Buring JE, Cook NR: Comparison of C-reactive protein and low-density lipoprotein cholesterol levels in the prediction of first cardiovascular events. N Engl J Med 2002, 347:1557-1565.

22. Ballantyne CM, Hoogeveen RC, Bang H, Coresh J, Folsom AR, Chambless LE, Myerson M, Wu KK, Sharrett AR, Boerwinkle E: Lipoprotein-associated phospholipase $\mathrm{A} 2$, high-sensitivity $\mathrm{C}$-reactive protein, and risk for incident ischemic stroke in middle-aged men and women in the Atherosclerosis Risk in Communities (ARIC) study. Arch Intern Med 2005, 165:2479-2484.

23. Ouellet V, Weisnagel SJ, Marois J, Bergeron J, Julien P, Gougeon R, Tchernof A, Holub BJ, Jacques H: Dietary cod protein reduces plasma Creactive protein in insulin-resistant men and women. J Nutr 2008, 138:2386-2391. 
24. Yamori $Y$, Hamashima $Y$, Horie $R$, Handa $H$, Sato M: Pathogenesis of acute arterial fat deposition in spontaneously hypertensive rats. Jpn Circ J 1975, 39:601-609.

25. Yamori $Y$, Horie $R$, Sato $M$, Fukase M: Hypertension as an important factor for cerebrovascular atherogenesis in rats. Stroke 1976, 7:120-125.

26. Murakami S, Yamagishi I, Asami Y, Ohta Y, Toda Y, Nara Y, Yamori Y: Hypolipidemic effect of taurine in stroke-prone spontaneously hypertensive rats. Pharmacology 1996, 52:303-313.

27. Murakami S, Nara $Y$, Yamori $Y$ : Taurine accelerates the regression of hypercholesterolemia in stroke-prone spontaneously hypertensive rats. Life Sci 1996, 58:1643-1651.

28. Mizushima S, Nara Y, Sawamura M, Yamori Y: Effects of oral taurine supplementation on lipids and sympathetic nerve tone. Adv Exp Med Biol 1996, 403:615-622.

29. Yokogoshi H, Mochizuki H, Nanami K, Hida Y, Miyachi F, Oda H: Dietary taurine enhances cholesterol degradation and reduces serum and liver cholesterol concentrations in rats fed a high-cholesterol diet. J Nutr 1999, 129:1705-1712.

30. Murakami S, Kondo Y, Toda Y, Kitajima H, Kameo K, Sakono M, Fukuda N: Effect of taurine on cholesterol metabolism in hamsters: up-regulation of low density lipoprotein (LDL) receptor by taurine. Life Sci 2002 , 70:2355-2366.

31. Yamori Y, Murakami S, Nara Y: Activated acyl coenzyme A-cholesterol acyltransferase as a possible cause of rapid arterial fat deposition in genetic hypertensive rat models for atherosclerosis. J Vasc Med Biol 1991, 3:268-270

32. Nakano A, Inoue N, Sato $Y$, Nishimichi N, Takikawa K, Fujita $Y$, Kakino A, Otsui K, Yamaguchi S, Matsuda H, Sawamura T: LOX-1 mediates vascular lipid retention under hypertensive state. J Hypertens 2000

33. Chiba $Y$, Ando $K$, Fujita T: The protective effects of taurine against renal damage by salt loading in Dahl salt-sensitive rats. J Hypertens 2002, 20:2269-2274.

34. Wang $L$, Yu YH, Zhang LG, Wang Y, Niu N, Li Q, Guo LM: Taurine rescues vascular endothelial dysfunction in streptozocin-induced diabetic rats: correlated with downregulation of LOX-1 and ICAM-1 expression on aortas. Eur J Pharmacol 2008, 597:75-80.

35. Kitamori K, Kobayashi M, Tsuchikura S, Hiraoka-Yamamoto J, Yasui N, Nara Y Ikeda K, Yamori Y, Nakajima T: High fat diet-induced liver injury and lipid metabolic disorder in arteriolipidosis-prone rat (ALR) [abstract]. Proceedings of the 2nd International Symposium on Lifestyle Related Diseases 2006, 33.

36. Yamori Y, Murakami S, Nara Y, Ikeda K: Stroke-prone SHR and arteriolipidosis-prone SHR as models for atherosclerosis: thei mechanisms and application for nutritional and pharmacological studies. Clin Exp Pharmacol Physiol Suppl 1995, 22:S244-245.

37. Yamori Y: Food factors for atherosclerosis prevention: Asian perspective derived from analyses of worldwide dietary biomarkers. Exp Clin Cardiol 2006, 11:94-98.

38. Yamori Y: Food culture: Development and Education. Paris: UNESCO 2009

39. Yamori $Y$, Liu L, Ikeda K, Mizushima S, Nara Y, Simpson FO: Different associations of blood pressure with 24-hour urinary sodium excretion among pre- and post-menopausal women. WHO Cardiovascular Diseases and Alimentary Comparison (WHO-CARDIAC) Study. J Hypertens 2001, 19:535-538.

40. Liu L, Mizushima S, Ikeda K, Nara Y, Yamori Y: Resting heart rate in relation to blood pressure: Results from the World Health OrganizationCardiovascular Disease and Alimentary Comparison Study. Int J Cardiol 2000

41. Palatini P, Julius S: Heart rate and the cardiovascular risk. J Hypertens 1997, 15:3-17

42. Okamura T, Hayakawa T, Kadowaki T, Kita Y, Okayama A, Elliott $P$, Ueshima H: Resting heart rate and cause-specific death in a 16.5-year cohort study of the Japanese general population. Am Heart J 2004 147:1024-1032

43. Sato $Y$, Ogata E, Fujita T: Hypotensive action of taurine in DOCA-salt ratsinvolvement of sympathoadrenal inhibition and endogenous opiate. Jpn Circ J 1991, 55:500-508

44. Mano M, Nara Y, Kihara M, Horie R, Endo J, Yamori Y: Enhanced urinary epinephrine excretion by cold exposure: A possible predictor of hypertension in rat models and humans. J Cardiovasc Pharmacol 1987, 10 S165-S166.
45. Mori M, Thorpe on behalf of Japan-Australian CARDIAC Group: Australian Aboriginals with highest risks and a new attempt for their nutritional improvement. Food Culture: Development and Education Paris: UNESCOYamori Y. 2009, 97-101.

46. O'Dea K: Marked improvement in carbohydrate and lipid metabolism in diabetic Australian aborigines after temporary reversion to traditional lifestyle. Diabetes 1984, 33:596-603.

47. Huxtable RJ: Physiological actions of taurine. Physiol Rev 1992, 72:101-163.

48. Huxtable RJ: Expanding the circle 1975-1999: sulfur biochemistry and insights on the biological functions of taurine. Adv Exp Med Biol 2000, 483:1-25.

49. Xu YJ, Saini HK, Zhang M, Elimban V, Dhalla NS: MAPK activation and apoptotic alterations in hearts subjected to calcium paradox are attenuated by taurine. Cardiovasc Res 2006, 72:163-174.

50. Ozcan U, Yilmaz E, Ozcan L, Furuhashi M, Vaillancourt E, Smith RO, Görgün CZ, Hotamisligil GS: Chemical chaperones reduce ER stress and restore glucose homeostasis in a mouse model of type 2 diabetes. Science 2006, 313:1137-1140.

51. Zulli A, Lau E, Wijaya BP, Jin X, Sutarga K, Schwartz GD, Learmont J, Wookey PJ, Zinellu A, Carru C, Hare DL: High dietary taurine reduces apoptosis and atherosclerosis in the left main coronary artery: association with reduced CCAAT/enhancer binding protein homologous protein and total plasma homocysteine but not lipidemia. Hypertension 2009, 53:1017-1022.

52. Imanishi T, Moriwaki C, Hano T, Nishio I: Endothelial progenitor cell senescence is accelerated in both experimental hypertensive rats and patients with essential hypertension. J Hypertens 2005, 23:1831-1837.

53. Hano $T$, Kasano H, Tomari N, Kuriyama T, Kawabe T: Taurine improves vascular tone through the suppression of peripheral sympathetic tone in endothelial progenitor cell senescence in hypertension [abstract]. Physiol Res 2008, 57:44-45.

54. Hano T, Kawabe T: Effect of taurine and estrogen on endothelial progenitor cell senescence in spontaneously hypertensive rats (SHR/ Izm). Clin Exp Hypertens 2000.

55. Fennessy FM, Moneley DS, Wang JH, Kelly CJ, Bouchier Hayes DJ: Taurine and vitamin $\mathrm{C}$ modify monocyte and endothelial dysfunction in young smokers. Circulation 2003, 107:410-415.

56. Yamori $Y$, Mizushima S: A review of the link between dietary magnesium and cardiovascular risk. J Cardiovasc Risk 2000, 7:31-35.

57. Touyz RM: Role of magnesium in the pathogenesis of hypertension. $\mathrm{Mol}$ Aspects Med 2003, 24:107-136.

58. Barbagallo M, Dominguez $L$, Resnick LM: Magnesium metabolism in hypertension and type 2 diabetes mellitus. Am J Ther 2007, 14:375-385.

59. Adachi M, Nara Y, Mano M, Ikeda K, Horie R, Yamori Y: Intralymphocytic free calcium and magnesium in stroke-prone spontaneously hypertensive rats and effects of blood pressure and various antihypertensive agents. Clin Exp Pharmacol Physiol 1993, 20:587-593.

60. Adachi M, Nara Y, Mano M, Yamori Y: Effect of dietary magnesium supplementation on intralymphocytic free calcium and magnesium in stroke-prone spontaneously hypertensive rats. Clin Exp Hypertens 1994, 16:317-326.

61. Hatzistavri LS, Sarafidis PA, Georgianos PI, Tziolas IM, Aroditis CP Zebekakis PE, Pikilidou MI, Lasaridis AN: Oral magnesium supplementation reduces ambulatory blood pressure in patients with mild hypertension. Am J Hypertens 2009, 22:1070-1075.

62. Shimosawa T, Takano K, Ando K, Fujita T: Magnesium inhibits norepinephrine release by blocking $\mathrm{N}$-type calcium channels at peripheral sympathetic nerve endings. Hypertension 2004, 44:897-902.

doi:10.1186/1423-0127-17-S1-S6

Cite this article as: Yamori et al:: Taurine in health and diseases: consistent evidence from experimental and epidemiological studies. Journal of Biomedical Science 2010 17(Suppl 1):S6. 